\title{
EFFECT OF DIFFERENT PREMOLAR ENDOCROWN PREPARATION DESIGNS ON THE TRUENESS OF DIFFERENT VIRTUAL IMPRESSION TECHNIQUES
}

\author{
Ghada Abdel Fattah*
}

\begin{abstract}
Purpose : The study was designed to evaluate the trueness of two premolar endocrowns preparation design with different virtual impression techniques

Material and methods: Two premolar teeth were endodontically treated, prepared using a CNC machine. Tooth A was prepared to have a flat occlusal surface, while tooth B was prepared to have a $45^{\circ}$ cusp anatomy inclination. For each tooth, a total of 40 different impressions were obtained. They were divided into 4 groups $(n=10)$ as follows: Group 1; direct scan using Medit i500, group 2; direct scan using trios 3shape, group 3; Putty and light impression were taken and impression was scanned using Identica Hybrid, group 4; Impressions were poured in gypsum and tooth model was scanned using identica hybrid. Both CAD reference models and all (STL) files $(n=80)$ were loaded into a 3D reverse engineering software (Geomagic QualifyTM 2012, Geomagic, Morrisville, USA). By using this method, for each superimposition, the deviation at each measurement point was recorded as a root mean square.
\end{abstract}

Results : There statistically significant differences between different groups. Anatomical reductions showed statistically significant higher trueness when compared to flat occlusal reduction. As for impression method, no statistically significant differences between direct Trios 3Shape scanning and impression scanning. However, both showed higher trueness when compared to direct Medit i500 scanning and cast scanning.

Conclusion : Anatomical preparation can be reproduced more accurately than flat preparation. Scanning direct impression produce more accurate virtual impression.

KEY WORDS: Endocrown preparations, premolar endocrowns, virtual impression technique

* Lecturer of Fixed Prosthodontics, Faculty of Dentistry, Ain Shams University 


\section{INTRODUCTION}

When restoring endodontically treated teeth with major loss of coronal hard tissue, a crown retained with post placed interradicular and core was the treatment of choice. Nowadays with the continuous search for less invasive restoration, the so- called endocrown gained more and more popularity among both clinicians and patients as a treatment alternative for treating the non-vital endodontically treated teeth. The endocrown preparation consist of circumferential butt margin and retained by engaging the whole pulp chamber and bonded with resin cement to the tooth structure. The restoration is most commonly fabricated using CAD/CAM technology. ${ }^{(1)}$.

Nowadays the concept of endocrowns have been extended to involve premolars and even incisors despite the debate regarding their biomechanical behaviour and long-term serviceability. There are no definite preparation guidelines in literature to guarantee the best biomechanical behavior. Many preparation attempts have been suggested especially concerning the occlusal surface to insure cuspal coverage and maximum fracture resistance of the restoration. ${ }^{(2)}$

The fabrication of a restoration using CAD/ CAM technology involves three main steps: the digital scanning of the abutment tooth or model, the designing of the restoration, and the actual milling of the crown. In the fabrication of restorations using $\mathrm{CAD} / \mathrm{CAM}$, long-term clinical success depends on the accuracy of the scanners, the designing software, and the milling machine, which in turn determines the accuracy of the restoration in terms of marginal and internal fit. ${ }^{(3)}$

Recently digitalizing the oral cavity and creating a three-dimensional virtual model technique has been introduced ${ }^{(1)}$. The virtual protocol consists of creating a scanning path for creating a virtual replica of the object. P. Muller and et al at 2016 ,reported that accuracy of the final virtual three dimensional model depend mainly on creating an accurate digital workflow starting from the scanning process ${ }^{(4)}$ therefore, choosing and creating the right scanning path is of prime importance.

Virtual impression techniques systems either uses direct digitalization techniques through different intraoral scanners or indirect digitalization technique represented by extraoral scanners. Intraoral scanners eliminate the need for a conventional impression, and its associated possible inaccuracies. Despite the fact that virtual casts obtained from intra oral scanners have high percentage of precision and accuracy, it is still limited with the various oral clinical conditions, specially when scanning complete arch casts, in this situation the accuracy of extraoral scanners still exceeds. Using extraoral scanners in dental labs become routine work nowadays, normally a master cast is poured from the impression and then scanned ,but with the advances in extraoral scanners technology it is now possible to scan the impression directly. ${ }^{(5)}$

Accuracy can be defined as the closeness of the measured value to a standard or a known (true) value. Precision is the closeness of measured values to each other. According to standards set by ISO 12836:2015 accuracy can be measured in terms of precision and trueness, where trueness is closeness of agreement between the mean obtained from repeated measurement and a true value. To determine the accuracy of a certain digital device, these standards are usually used. The higher the trueness of a certain device means its higher ability to reproduce the object actual dimension. Moreover, when the device obtain repeated consistent measures or scan it is safe to say it has high precision.. ${ }^{(6)}$

Therefore, trueness of a virtual impression technique can be detected by the divergence of the scanned impression from the original dimensions of the object to be tested, and its precision is the variation between the scans within each set tested. ${ }^{(7)}$

In order to be able to detect the difference in accuracy between virtual and conventional 
impression technique it is important to set a true value, a standard to refer to. This can be achieved by obtaining a surface tessellation language (STL) dataset of the object to be tested using a reference scanner, then comparing each technique trueness to this standard (true value). The comparison can be made by a software that superimposes each scan with the standard one separately using a best fit algorithm. ${ }^{(8)}$

Regardless of the method used, $120 \mu \mathrm{m}$ marginal gap is the clinically accepted gap value of restorations. ${ }^{(9)}$

Thus, this study was conducted to evaluate the trueness of the intraoral scanning compared to extraoral scanning directly from impression or indirectly from a poured cast, within two different occlusal reduction preparation for premolars endocrowns.

Two null hypotheses were suggested there will be no difference between virtual impression techniques and the second no difference between flat or anatomical occlusal preparation.

\section{MATERIALS AND METHODS}

Two sound caries-free upper first premolars extracted for orthodontic purpose from the same patient were selected. They were selected to be within the following range of dimensions (9-10 $\mathrm{mm}$ bucco-palatal dimension, 7-8 $\mathrm{mm}$ mesio-distal dimension and pulp chamber depth ranged from 5-7 $\mathrm{mm}$ from central groove).

For standardization purpose, both teeth were endodontically treated with the same sequence and by the same operator. The pulp chamber was accessed following its own pulp chamber morphology via a round carbide high speed bur. Canal lengths were determined visually by-passing size \#10 K-file through the root canals until being obvious at the apical foramen, working lengths were adjusted $1 \mathrm{~mm}$ short from apical foramen.
Protaper system (Dentsply-Maillefer; Switzerland) was used for root canals treatment for standardization following the manufacturer instruction, F2 were used as master file for both canals, sodium hypochlorite was used as an irrigant after each used file. Protaper paper points and gutta percha size F2 were used. Resin based root canal sealant was used and then a heated condenser and a plugger were used for removal of the excess gutta percha till the canal orifice.

Both teeth were then mounted in a polypropylene mold and prepared using a CNC machine to standardize preparation dimensions and have $8^{\circ}$ divergence throughout internal walls. Tooth A was prepared to have a flat occlusal surface and $4 \mathrm{~mm}$ intrapulpal depth, while tooth $\mathrm{B}$ was prepared to have a $45^{\circ}$ cusp anatomy inclination and $4 \mathrm{~mm}$ intrapulpal depth.

To create a standard reference file each tooth was scanned using an extra-oral desktop scanner (Identica Hybrid, Medit, Seoul, Korea) and a STL file was exported. For each tooth, a total of 40 different impressions were obtained. They were divided into 4 groups $(\mathrm{n}=10)$ as follows: Group 1; direct scan using Medit i500 (MEDIT corp., Seoul, Korea), group 2; direct scan using trios 3shape (3Shape A/S, Copenhagen K Denmark), group 3; Putty and light impression were taken and impression was scanned using Identica Hybrid, group 4; Impressions were poured in gypsum and tooth model was scanned using Identica hybrid. For each tooth a total of 40 STL files were generated.

Both CAD reference models and all (STL) files $(n=80)$ were loaded into a 3D reverse engineering software (Geomagic QualifyTM 2012, Geomagic, Morrisville, USA) and all unnecessary information were cut using the "cut with planes" function. For the trueness measurement, color difference maps and reports $(\mathrm{n}=80)$ were obtained by superimposing the digital data sets of the scans onto the reference model, then (3D) deviation analysis was performed 


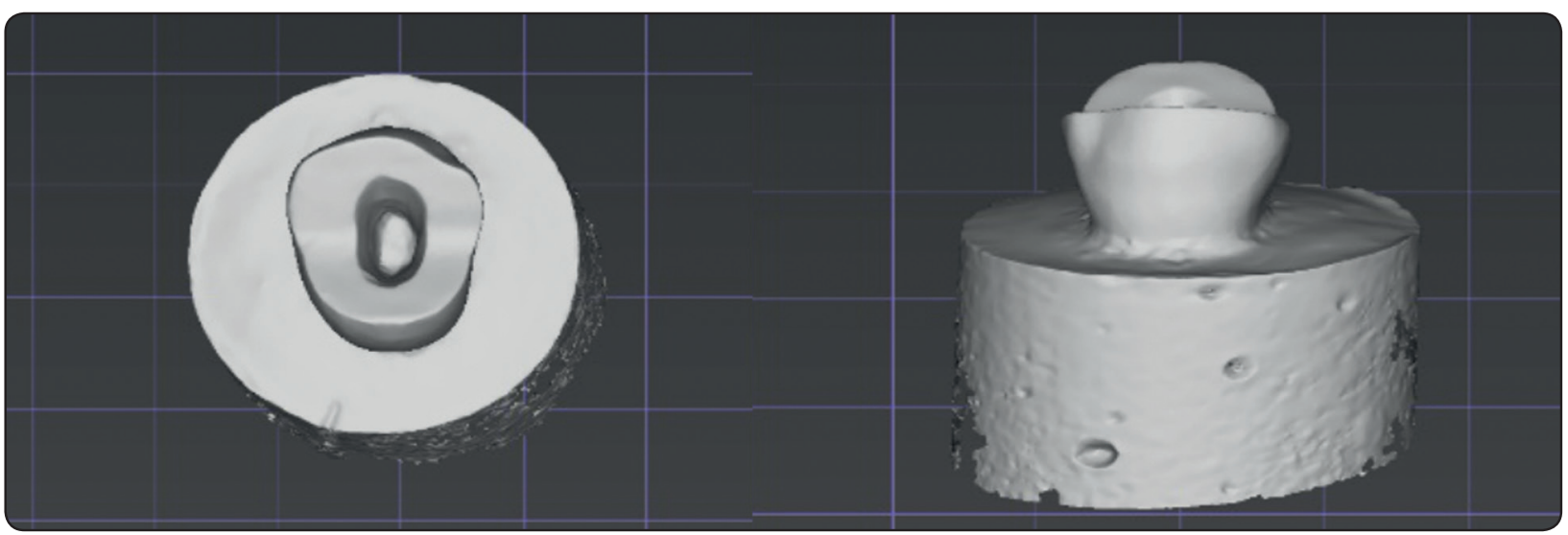

Fig. (1) Showing scanned die

with the best-fit algorithm method. By using this method, for each superimposition, the root mean square (RMS) of the amount of deviation at each measurement point was recorded.

The data collected was checked for normal distribution and analyzed using two-way analysis of variance(ANOVA),followed by Tukey'sHSD test (SPSS v20, Chicago, IL, USA) at a significance level of $\mathrm{P} \leq 0.05$.

\section{RESULTS}

Mean values and standard deviations (SD) of trueness measured in micrometers $(\mu \mathrm{m})$ of all groups are listed in Table I.

Two-way ANOVA tests showed significant differences between different groups. Anatomical reductions showed statistically significant higher trueness when compared to flat occlusal reduction.
As for impression method, Tukeys post-hoc revealed no significant differences between direct Trios 3Shape scanning and impression scanning. However, both showed higher trueness when compared to direct Medit i500 scanning and cast scanning.(fig1)

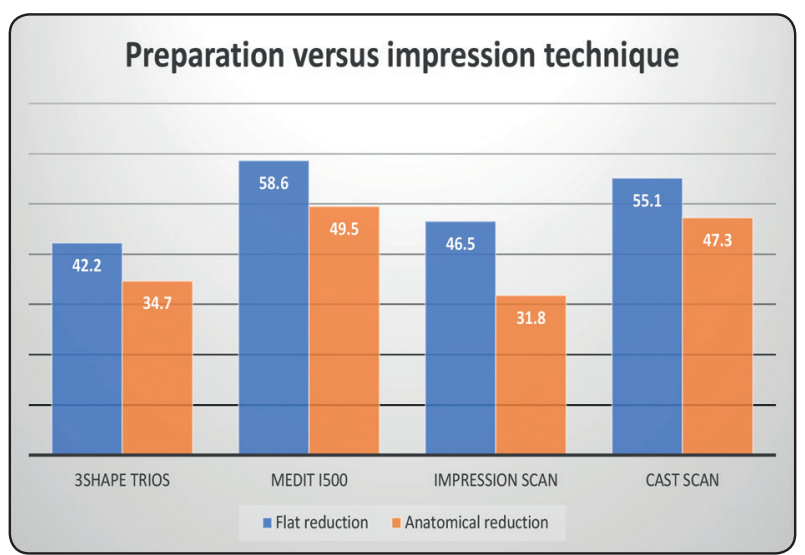

Fig. (1) Graph representing mean of all tested groups in micrometres.

TABLE (I) Table showing mean and standard deviation of trueness value of all tested groups

\begin{tabular}{|c|c|c|c|c|c|c|c|c|}
\hline Occlusal Surface & \multicolumn{4}{|c|}{ Flat reduction } & \multicolumn{3}{c|}{ Anatomical reduction } \\
\hline $\begin{array}{c}\text { Impression } \\
\text { technique }\end{array}$ & 3Shape Trios & Medit i500 & $\begin{array}{c}\text { Impression } \\
\text { Scan }\end{array}$ & Cast Scan & $\begin{array}{c}3 \text { Shape } \\
\text { Trios }\end{array}$ & Medit i500 & $\begin{array}{c}\text { Impression } \\
\text { Scan }\end{array}$ & Cast Scan \\
\hline $\begin{array}{c}\text { Mean }(\text { SD) RMS } \\
\text { in } \mu \mathrm{m}\end{array}$ & $42.2(3.1)^{\mathrm{a}}$ & $58.6(3.7)^{\mathrm{b}}$ & $46.5(4.3)^{\mathrm{a}}$ & $55.1(2.9)^{\mathrm{b}}$ & $34.7(3.2)^{\mathrm{c}}$ & $49.5(4.7)^{\mathrm{d}}$ & $31.8(4.1)^{\mathrm{c}}$ & $47.3(3.9)^{\mathrm{ad}}$ \\
\hline
\end{tabular}

Different superscript letters denotes statistically significant means 


\section{DISCUSSION}

Dental impressions aim to meticulously imitate the intraoral condition of the patient and passing this detailed information to the master cast. Obtaining a master cast with high trueness is crucial for the final treatment plan success; to achieve this purpose a variety of materials and techniques have been used to reach an impression with desired accuracy. ${ }^{(10)}$

With the continuous search for accuracy and seeking solution for problems in conventional impression techniques the evolution of the virtual digital impression became a must. This evolution mainly depends on reproducing the intra oral condition via three-dimensional system creating a virtual model where the final restoration can be fabricated. ${ }^{(11)}$

Both tooth and restoration type affect the ability of the digital scanner to accurately reproduce the oral cavity., ${ }^{(12)}$ thus this study was conducted to evaluate the complex endo crown premolar preparation with variable occlusal morphology in order to define a better scanning method to achieve more accurate restoration.

For eliminating inter -operators' errors only one experienced operator accustomed with all scanning devices used in the study preformed the scans. A desktop scanner (Identica) was used as a reference scanner to create digital reference data as intraoral scanners has always been corelated high degree of movement freedom during scanning, this movement usually result in more and more errors caused by abrupt actions during scanning leading to the bending of the model. ${ }^{(13)}$

Human natural teeth have been selected in this study. Artificial abutments might provide standardized preparations and identical physical qualities of materials used in comparison to natural teeth, however the later ensure more simulation to clinical conditions with respect to tooth architecture and morphology. The dentin and enamel surfaces for bonding, the contour of the pulp chamber and root canals, and the ratio between the crown and root are more accurate and clinically reliable than on artificial teeth. ${ }^{(14)}$

Four different types of digital impressions techniques were chosen two intraoral scanners, one representing still images with stitching techniques (3 Shape Trios), video imaging (Medit i 500) and extraoral scanners (Identica):scanned directly from impression and then from stone cast poured from the impression.

Although results were significantly different between all tested groups but all results were within the clinically acceptable marginal gap for restorations $120 \mu \mathrm{m} .{ }^{(9)}$

Using image superimposition method for evaluation is challenging, it has fundemetal errors and limitation specially in selecting randomized superimposition points on the program. These points might or might not represent the actual restoration. But still it is the method of choice in comparing virtual impression techniques. ${ }^{(13)}$

To obtain a virtual impression we can either use intraoral or extraoral scanner, which, like an ordinary camera, collects information about projecting light. Reproducible tissues are shown on the hardware display as natural looking. The intraoral scanner measures the light reflection times of the subject surface. The description, based on data and calculation algorithms to copy the software, calculates, and generates a computer screen image of the prepared area. ${ }^{(15)}$

Both null hypotheses were rejected. The result of this study in relevance to type of preparation reduction showed that the anatomical reductions have statistically significant higher trueness when compared to flat occlusal reduction.

The accuracy of intraoral scanners depends on the position of the tooth in the dental arch, where it shows better accuracy in posterior than anterior teeth, this can be attributed to the presence of more anatomical configuration in posterior teeth. The tooth shapes (steep inclination of cusps and depth 
of fissures, flat teeth) might have an influence on the scanner accuracy ${ }^{(16)}$.

In general, Intraoral scanners cause a horizontal displacement, because they transmit a light source from the end of a small head and stitch the scanned area through a small scan range, the trueness of intra oral scanners was lower in the fixed dental prosthesis with complex details, which can be attributed to the errors in the image acquisition and stitching processes due to the presence less surface characteristics like flat surface preparations, but still the video acquisition showed more accurate results in flat surfaces as images can be attentively monitored during scanning. Although extraoral scanners is also affected by the tooth type, it generally shows less divergence in comparison ${ }^{(17)}$.

This explains the different in results found between scanning the flat and the anatomical preparation.

As for virtual impression technique, although there were no significant differences between direct Trios 3Shape scanning and impression scanning. However, both showed higher trueness when compared to direct Medit i500 scanning and cast scanning. This can be attributed to different digital impression technology used as well as the complexity of the preparation scanned.

Virtual intra oral techniques vary according to the mode of image acquisition it can either be video or still photos. Still photos depends on triangulation or parallel confocal laser scanning in which a series of images are obtained and then stitched together to form a $3 \mathrm{D}$ reconstructive image. These images are easier and more accurately stitched together in the presence of more complex dental anatomy. This complex geometrical anatomy are more commonly found in the occlusal surface of molars and premolars which makes them more easier to stich than flat anterior tooth geometry, where video imaging showed better results. This explains the slight differences in results among the two intraoral scanners used with the different preparation although it was statistically insignificant. ${ }^{(18)}$ The aforementioned facts affect the accuracy of the final virtual impression which can be easily reflected on the fit of the final restoration.

The i500 (Medit) IOS was introduced in 2018, and therefore, the literature regarding its accuracy is limited. In vitro complete-arch precision values for this scanner range from 52.3 to $66.3 \mathrm{~mm}$ coinciding with those reported in this study. ${ }^{(19)}$

Reflection laws states, the angle of incident rays equals that of reflected rays to the normal at the point of reflection. Surface properties of material, such as translucency, matte, and porous surfaces and added added properties from coating will create different microscopic planes in the three dimensions. This will cause the incident rays to scatter, resulting in a diffuse reflection. ${ }^{(20)}$ So when using different scanned surface (impression material and gypsum models) it is expected to have different results,

Moreover, the distortion factors of indirect digitization are well explored according to the conventional impression techniques, impression materials, pouring techniques, gypsum materials and sectioning systems. ${ }^{(21)}$ According to ADA Specification\#25 crystallization of gypsum results in residual stresses causing up to $0.2 \%$ expansion $^{(22)}$. So virtual model obtained directly from pouring of gypsum cast showed the least trueness among tested groups even when using extraoral scanners.

Major limitation of the current study was its in vitro design. Mimicking the oral cavity situation with the complete arch, saliva, patient movement and accessing posterior teeth was not totally reproduced. The aforementioned conditions affects the accuracy of the virtual impression specially the intraoral one. ${ }^{(23)}$

Preparation with more complex details are nowadays more currently practiced, virtual scanning is becoming more and more popularly used so the search for which virtual technique is better with which restoration should be continued. 


\section{CONCLUSIONS}

Taking into consideration the limitation of the study we can conclude the following:

- Digital impression techniques are more accurate when scanning anatomical preparation

- Flat preparation is better scanned with video scanning technique

- Scanning impression directly by desktop scanners is the most effective digital impression technique.

\section{RREFERENCES}

1. Bernhart J, Bräuning A, Altenburger MJ, Cerec3D endocrowns-two-year clinical examination of CAD/CAM crowns for restoring endodontically treated molars. Int $\mathrm{J}$ Comput Dent 2010;13:141-154.

2. Serin Kalay T, Yildirim T, Ulker M. Effects of different cusp coverage restorations on the fracture resistance of endodontically treated maxillary premolars. Journal of Prosthetic Dentistry 2016; 116: 404-10.

3. Mai Salaheldin, Ashraf Hussein Sherif, Ahmed Naguib Mohammedand Shereen Ahmed Nossair. Comparison of Accuracy of Restorations Generated Through Digital Images of Two Extra Oral Scanners. E.D.J. Vol. 65, No. 3 July, 2019.

4. P. Muller, A. Ender, T. Joda, and J. Katsoulis, “. Impact of digital intraoral scan strategies on the impression accuracy using the TRIOS Pod scanner,". Quintessence International, vol. 47, pp. 343-349, 2016.. .

5. Guth JF, Keul C, Stimmelmayr M, Beuer F, Edelhoff D. Accuracy of digital models obtained by direct and indirect data capturing. Clin Oral Investig. 2013 May; 17(4): 1201-8.

6. ISO 12836. Dentistry-Digitizing Devices for CAD/CAM Systems for Indirect Restorations-Test for Methods for Assessing Accuracy,. International Organization for Standardization, Geneva, Switzerland, 2015.

7. Ender A, Mehl A: In-vitro evaluation of the accuracy of conventional and digital methods of obtaining full-arch dental impressions. Quintessence Int 2015;46:9-17 .

8. Ahlholm P, Sipila K, Vallittu P, Jakonen M, Kotiranta U. Digital versus conventional impressions in fixed prosthodontics: a review. J Prosthodont. 2016.
9. McLean JW, von Fraunhofer JA: The estimation of cement film thickness by an in vivo technique. Br Dent J 1971; 131:107-111.

10. Arag. N, M. L., et al. Validity and reliability of intraoral scanners compared toconventional gypsum models measurements: a systematic review. Eur. J. Orthod., 38(4): 429-34, 2016.

11. Christensen, G. J. Impressions are changing: deciding on conventional, digital or digital plus in-office milling. Impressions are changing: deciding on conventional, digital or digital plus in-office milling. J. Am. Dent. Assoc., 140(10):1301-4, 2009.

12. Keunbada Son and Kyu-bok Lee. Effect of Tooth Types on the Accuracy of Dental 3D Scanners: An In Vitro Study. . Materials 2020, 13, 1744.

13. George Michelinakis, Dimitrios Apostolakis, Andreas Tsagarakis, George Kourakis, Emmanuil Pavlakis,. A comparison of accuracy of 3 intraoral scanners: A singleblinded in vitro study. j.prosdent 2019 Dec 21;S00223913(19)30703-6.

14. Nawrocka A, Lukomska-Szymanska M. Extracted human teeth and their utility in dental research. Recommendations on proper preservation: A literature review. . Dent Med Probl 2019; 56: 185-90.

15. Dahl BE, Ronold HJ. Digital impressions. . Finnish Dent J 2014;4:32-38.

16. Park, J.M., Kim, R.J.Y. and Lee, K.W. Comparative reproducibility analysis of 6 intraoral scanners used on complex intracoronal preparations. . J. Prosthet. Dent. 2020, 123, 1.

17. Park, Ji-Man. Comparative analysis on reproducibility among 5 intraoral scanners: sectional analysis according to restoration type and preparation. . J Adv Prosthodont 2016;8:354-62.

18. Pekka Ahlholm, Kirsi Sipilä, Pekka Vallittu, Minna Jakonen, \& Ulla Kotiranta,. Digital Versus Conventional Impressions in Fixed Prosthodontics: A Review . Journal of Prosthodontics 27 (2018) 35-41.

19. Ender A, Zimmermann M, Mehl A. Accuracy of completeand partial-arch impressions of actual intraoral scanning systems in vitro. Int J Comput Dent 2019;22:11-9.

20. Gursharan Kaur Sason, Gaurang Mistry, Rubina Tabassum, Omkar Shetty. A comparative evaluation of intraoral and extraoral digital impressions: An in vivo study. The Journal of Indian Prosthodontic Society I Volume 18 I Issue 2 | Apr-June 2018. 
21. Lee K-T, Kim H-Y, Kim W-C, Kim J-H, Jeon J-H. White light scanner-based repeatability of 3-dimensional digitizing of silicon rubber abutment teeth impressions. . J Adv Prosthodont. 2013;5(4):452.

22. Council on Dental Materials. Instruments, and Equipment.
Revised ANSI/ ADA specification no. 2. J Am Dent Assoc 1985; 111(6):1003.

23. Goracci C, Franchi L, Vichi A, Ferrari M. Accuracy, reliability, and efficiency of intraoral scanners for full-arch impressions: a systematic review of the clinical evidence. Eur J Orthod 2016;38:422-8. 